\title{
BULLOUS SYSTEMIC LUPUS ERYTHEMATOSUS - A CASE REPORT
}

\author{
Ali $\mathrm{CM}^{1}$, Kamal $\mathrm{SMB}^{2}$, Khan $\mathrm{MMH}^{3}$
}

\begin{abstract}
Bullous systemic lupus erythematosus (BSLE) is a rare but distinct disease, characterized by vesicobullous skin eruptions in systemic lupus erythematosus (SLE). It can arise either before or after a diagnosis of SLE has been established. BSLE is characterized by a dermatitis herpetiformis-like histology and an autoimmunity to type VII collagen. It must be differentiated from other autoimmune vesicobullous diseases such as epidermolysis bullosa acquisita, dermatitis herpetiformis, linear IgA disease, and bullous pemphigoid. A combination of clinical, histological, and immunofluorescence findings are necessary to establish a diagnosis of BSLE. We present a case of BSLE to illustrate and emphasize the need for an integrative diagnostic approach.
\end{abstract}

Key words: Bullous systemic lupus erythematosus, systemic lupus erythematosus, autoimmune disease.

J Dhaka Med Coll. 2013; 22(1) : 90-92.

\section{Introduction}

Bullous systemic lupus erythematosus (BSLE) is a rare dermatosis that is regarded as a distinct variant of systemic lupus erythematosus (SLE)1-4. It is characterized by widespread vesicobullous eruptions, a dermatitis herpetiformis-like histology, immunologic features resembling epidermolysis bullosa acquisita (EBA), and striking response of cutaneous lesions to dapsone treatment. We present a case of BSLE to illustrate and emphasize the need for an integrative diagnostic approach, to include clinical, histological, and immunofluorescence findings.

\section{Case Report}

A 25 years old, married female presented with multiple tense blisters on her face, neck, front and back of the trunk, both upper \& lower extremities. These lesions are eventually ruptures and healed up spontaneously without scarring leaving multiple hypopigmented patches. She also complains of oral ulceration but no difficulty in swallowing. Her parents and siblings have no similar disease. She has no pruritus. She has no history of trauma induced blistering. Oral mucosa reveals a small ulceration on erythematous base in buccal mucosa. Hairs, nails are normal. Examination of other system reveals no abnormalities. The patient was ANA and Anti-ds DNA positive. Histopathological examination reveals subepidermal bulla with eosinophils and some neutrophils. DIF shows linear deposition of IgG and $\mathrm{C} 3$ in the basement membrane zone. We treated the patient with systemic steroid and dapsone and got good response.

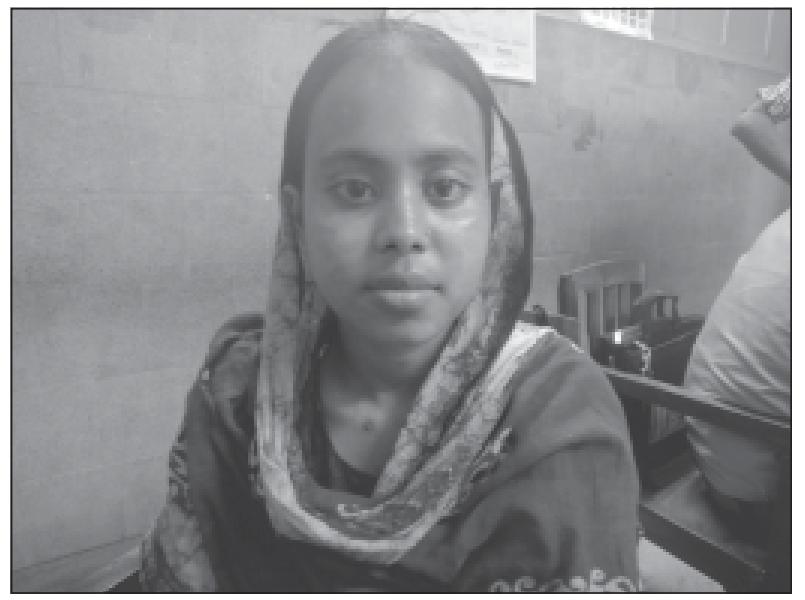

Fig.-1: Before treatment (face).

1. Dr. Chowdhury Mohammad Ali, Professor \& Head, Department of Skin \& VD, Dhaka Medical College, Dhaka.

2. Dr. SM Bakhtiar Kamal, Assistant Professor, Department of Skin \& VD, Dhaka Medical College, Dhaka.

3. Dr. Md. Mosaraf Hossain Khan, Registrar, Department of Nephrology, Dhaka Medial College, Dhaka.

Correspondence: Dr. Chowdhury Mohammad Ali, Professor \& Head, Department of Skin \& VD, Dhaka Medical College, Dhaka. 


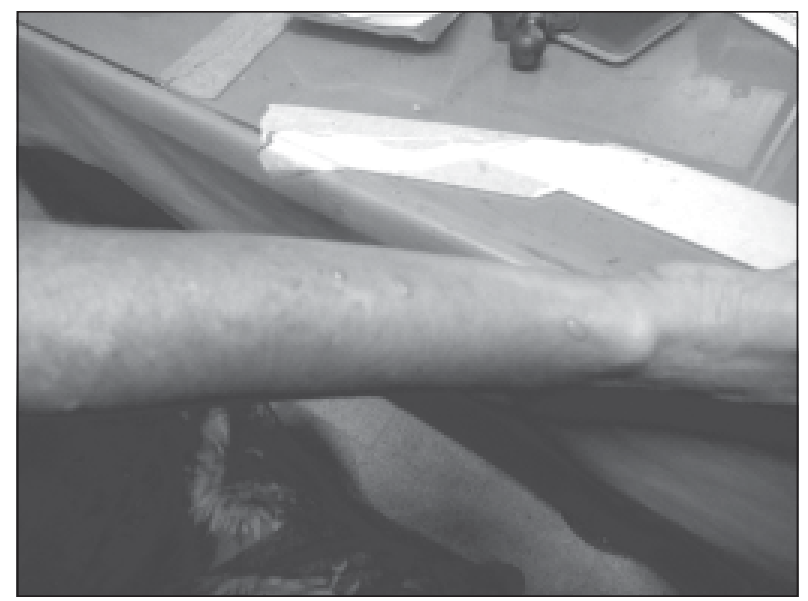

Fig.-2: Before treatment (hands).

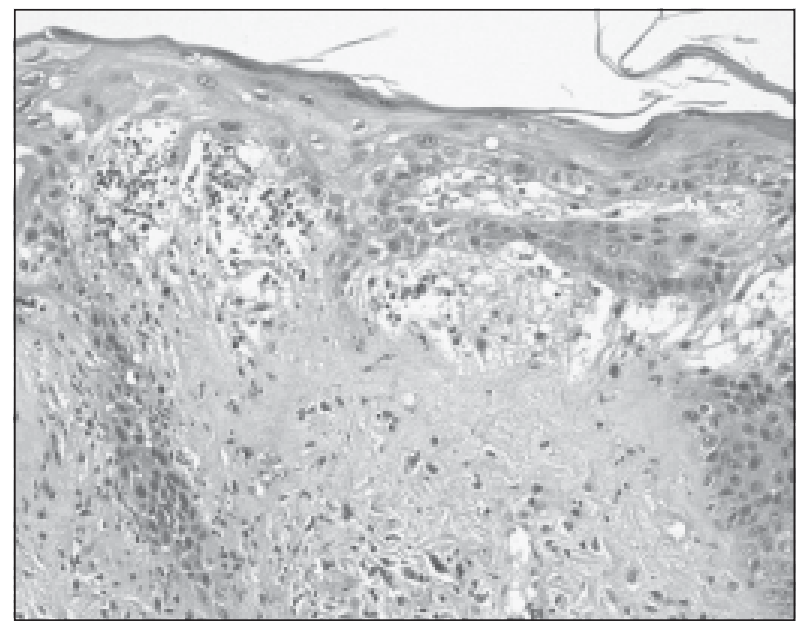

Fig.-4: Histopathological slide

\section{Discussion}

Bullous SLE is an extremely uncommon subset of systemic lupus erythematosus with an incidence of 0.26 per million population per year in adults7. Patients with SLE rarely (in less than $1 \%$ ) develop widespread vesicobullous eruptions that cannot be classified as either of the primary bullous dermatoses and are not merely a manifestation of extreme basal cell hydropic degeneration and the resulting epidermal- dermal separation. In such cases, it is referred to as BSLE, a unique dermatosis that is regarded as a distinct variant of SLE14. It is characterized by dermatitis herpetiformis-like histology, immunologic features resembling EBA, and a striking response of cutaneous lesions to dapsone

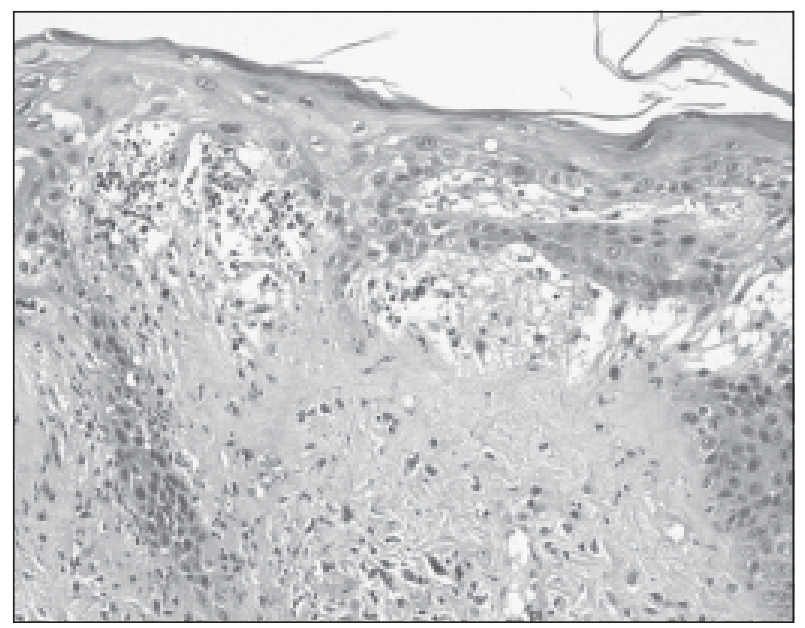

Fig.-3: Before treatment (leg).

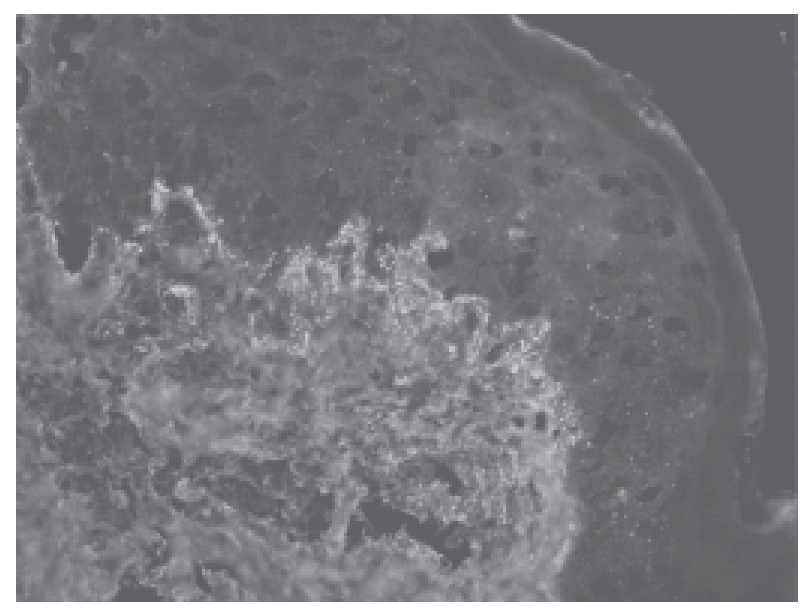

Fig.-5: DIF study

treatment. Similarly to SLE, BSLE most often affects young patients, predominantly female, in the second to fourth decades of life. Although any sun-exposed or non-sun-exposed areas can be affected, the upper trunk, neck, supraclavicular areas, and proximal extremities are predilection sites ${ }^{1,2}$. The skin eruption comprises bullae arising either from areas affected by diffuse erythema or on an urticarial base resembling bullous pemphigoid, or as grouped vesicular lesions mimicking dermatitis herpetiformis ${ }^{1,2,4}$. The onset of vesicobullous cutaneous lesions can precede, coincide with, or follow the diagnosis of SLE, as defined by the revised American Rheumatism Association criteria for classification of $\mathrm{SLE}^{3,5}$. Our patient met several diagnostic criteria: oral 
ulcers and a positive result for antinuclear $\&$ Anti-ds antibodies. Bullous changes in lupus erythematosus have two basic histological patterns: neutrophilic and mononuclear ${ }^{6}$. The neutrophilic pattern typical of BSLE is characterized by subepidermal vesicles or bullae containing fibrin, mixed inflammatory cell infiltrate with a predominance of neutrophils, and nuclear debris. These histological features simulate dermatitis herpetiformis or linear IgA disease ${ }^{1}$. In contrast to dermatitis herpetiformis and linear IgA disease, BSLE frequently includes the deposition of mucin in the dermis. On the other hand, bullous changes with a mononuclear inflammatory pattern can develop in the cutaneous lesions of lupus erythematosus as a result of extensive damage to the epidermal basal layer. This is due to intense interface dermatitis, which can result in epidermaldermal separation and vesicle formation. Such bullous changes, which can clinically resemble erythema multiforme or toxic epidermal necrolysis, represent a spectrum of changes resulting from cutaneous lupus erythematosus and are not referred to as BSLE ${ }^{1,6}$. Patients with SLE can produce a myriad of auto antibodies, resulting in diverse clinical manifestations.

Similarly to EBA, the pathogenesis of BSLE is associated with auto antibodies reacting with collagen type $\mathrm{VII}^{2,4}$. Multiple classes of immunoglobulins are often detected along the basement membrane by direct immunofluorescence: IgG has been found in nearly $100 \%$ of patients with BSLE, IgA in about $70 \%$, and IgM in $50 \%$. Deposition of immunoreactants along the basement membrane has been reported granular in $40 \%$ of cases, similar to the lupus band in non-bullous cutaneous lupus, or linear in $60 \%$, similar to the deposits in bullous pemphigoid or $\mathrm{EBA}^{1,3}$. In addition to deposits along the basement membrane, deposition of immunoreactions within vessel walls can be identified in $\mathrm{BSLE}^{1}$. The differential diagnosis of BSLE includes other autoimmune vesicobullous diseases such as EBA, dermatitis herpetiformis, linear IgA disease, and bullous pemphigoid ${ }^{1,3-6}$. EBA generally occurs in older patients than does BSLE, and it is associated with the formation of bullae at the sites of previous trauma or mechanical rubbing. These bullae heal with scarring and do not respond to dapsone treatment. Despite similar histological features, dermatitis herpetiformis can be distinguished from BSLE by observing granular deposits of IgA in dermal papillae ${ }^{3}$. Linear IgA dermatosis is characterized by the linear deposition of IgA along the basement membrane in perilesional skin ${ }^{5}$.

\section{References:}

1. McKee PH, Calonje E, Granter SR. ed. Pathology of the skin with clinical correlations. Vol.1. 3rd ed. Philadelphia: Elsevier Mosby; 2005.

2. Vassileva S. Bullous systemic lupus erythematosus. Clinics Dermatol. 2004; 22: 12938 .

3. Yell JA, Wojnarowska F. Bullous skin disease in lupus erythematosus. Lupus 1997; 6: 112-21.

4. Crowson NA, Magro C. The cutaneous pathology of lupus erythematosus: a review. J Cutan Pathol 2001; 28: 1-23.

5. Tan EM, Cohen AS, Fries JF, et al. The 1982 revised criteria for the classification of systemic lupus erythematosus. Arthritis Rheum 1982; 25: $1271-7$.

6. Elder DE. ed. Lever's Histopathology of the Skin. 10th ed. Philadelphia: Lippincott Williams \& Wilkins; 2009.

7. Wong SN, Chua SH. Spectrum of subepidermal immunobullous disorders seen at the National Skin Centre, Singapore: a 2-year review. Br J Dermatol 2002; 147: 476-80. 